\title{
Fermi Large Area Telescope observation of high-energy solar flares: constraining emission scenarios
}

\author{
Nicola Omodei ${ }^{1}$, Melissa Pesce-Rollins ${ }^{1,2}$, Vahè Petrosian ${ }^{1}$, Wei Liu ${ }^{1}$, \\ Fatima Rubio da Costa $^{1}$ and Alice Allafort ${ }^{1}$, for the Fermi-LAT \\ collaboration \\ ${ }^{1}$ Stanford University and KIPAC \\ email: nicola.omodei@stanford.edu \\ ${ }^{2}$ INFN Pisa, Italy
}

\begin{abstract}
The Fermi Large Area Telescope (LAT) is the most sensitive instrument ever deployed in space for observing gamma-ray emission $>100 \mathrm{MeV}$. This has also been demonstrated by its detection of quiescent gamma-ray emission from pions produced by cosmic-ray protons interacting in the solar atmosphere, and from cosmic-ray electron interactions with solar optical photons. The Fermi-LAT has also detected high-energy gamma-ray emission associated with GOES M-class and X-class solar flares, each accompanied by a coronal mass ejection and a solar energetic particle event, increasing the number of detected solar flares by almost a factor of 10 with respect to previous space observations. During the impulsive phase, gamma rays with energies up to several hundreds of $\mathrm{MeV}$ have been recorded by the LAT. Emission up to GeV energies lasting several hours after the flare has also been detected by the LAT. Of particular interest are the recent detections of three solar flares whose position behind the limb was confirmed by the STEREO satellites. While gamma-ray emission up to tens of $\mathrm{MeV}$ resulting from proton interactions has been detected before from occulted solar flares, the significance of these particular events lies in the fact that these are the first detections of $>100 \mathrm{MeV}$ gamma-ray emission from footpoint-occulted flares. We will present the Fermi-LAT, RHESSI and STEREO observations of these flares and discuss the various emission scenarios for these sources.
\end{abstract}

Keywords. Sun: flares, Sun: X-rays, gamma rays

\section{Fermi Large Area Telescope observation of solar flares}

Launched in 2008, the Fermi observatory is comprised of two instruments; the Large Area Telescope (LAT) designed to detect $\gamma$-rays from $20 \mathrm{MeV}$ up to more than 300 GeV (Atwood et al. 2009) and the Gamma-ray Burst Monitor (GBM) which is sensitive from $\sim 8 \mathrm{keV}$ up to $40 \mathrm{MeV}$ (Meegan et al. 2009). During the first 18 months of operation coinciding with the solar cycle minimum, the Fermi-LAT detected $>100 \mathrm{MeV}$ gammaray emission from the quiescent Sun (Abdo et al. 2011a). As the solar cycle approaches its maximum, the LAT has detected several solar flares above $30 \mathrm{MeV}$ during both the impulsive and the temporally extended phases (Ackermann et al. 2012, 2014; Ajello et al. 2014). The brightest flare observed so far by the LAT is the flare of 2012 March 07. The Fermi-LAT $>100 \mathrm{MeV}$ count rate was dominated by the gamma-ray emission from the Sun†, which was nearly 100 times brighter than the Vela Pulsar in the same energy range. The impulsive phase (the first eighty minutes) was followed by a long-lasting gamma-ray emission $(\sim 20 \mathrm{hr})$, whose maximum was delayed by several minutes from the impulsive

$\dagger$ http://apod.nasa.gov/apod/ap120315.html 


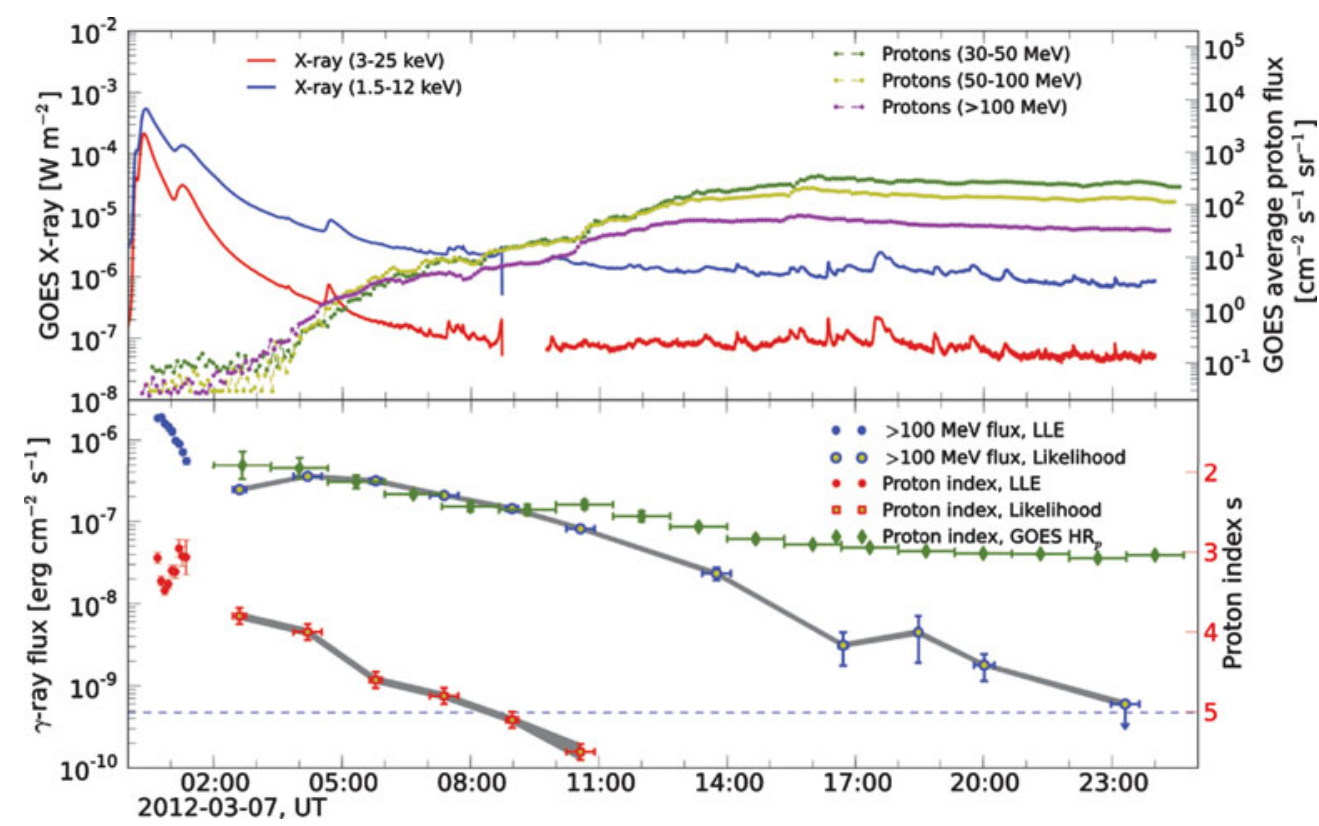

Figure 1. Impulsive and sustained emission of the 2012 March 7 flare. Top panel: soft X-rays (red: 3-25 keV, blue: 1.5-12 keV) from the GOES 15 satellite. On the right axis, 5-minute averaged proton flux (green: 30-50 MeV, yellow: 50-100 MeV, magenta: >100 MeV). Bottom panel: high-energy gamma-ray flux above $100 \mathrm{MeV}$ measured by the Fermi-LAT. The Blue/red circles represent the flux and the derived proton spectral index during the impulsive phase. The blue circles/red squares represent the flux and derived proton spectral index obtained by standard likelihood analysis during the sustained emission phase. Green diamonds are the GOES proton spectral indexes derived from the hardness ratio, as described in Ajello et al. (2014). The gray bands correspond to the systematic uncertainties on the effective area of the instrument. The horizontal dashed line corresponds to the value of the gamma-ray flux from the quiescent Sun, from Abdo et al. (2011b).

emission. In both episodes, the flux was well described by a pion decay spectrum from a power law proton distribution.

In the lower panel of Figure 1 the evolution of both the gamma-ray flux and the derived spectral index $s$ of the protons are shown: the impulsive and the sustained emission phases can be easily identified. Interestingly, unlike during the impulsive phase, the spectrum of the temporally extended emission becomes softer monotonically. In the top panel we report the evolution of the X-ray flux as well as the evolution of the proton spectrum detected by the GOES satellite. While, during the impulsive phase, the gamma-ray spectrum is correlated with the X-ray emission, during the sustained phase the gammaray emission appears to be more correlated with the proton flux, suggesting that the accelerated particles that produce $\gamma$-rays are probably related to the Solar Energetic Particles (SEP) detected at Earth.

\section{The Behind-the-limb flares detected by the Fermi-LAT}

Gamma-ray emission from solar flares is generally believed to occur predominantly in compact high-density regions near the magnetic footpoints. Observations of gamma-ray emission from flares whose active region (AR) is located behind the visible solar disk pose interesting questions regarding the interaction point and the transport of the accelerated particles during these rare events. During solar cycles 21 and 22, three gamma-ray behind- 
Table 1. Behind-the-limb flare properties. The start time of the flare is estimated from STEREO imaging. The GOES class is evaluated using STEREO-observed extreme ultraviolet intensity, as described by (Nitta et al. 2013). The CME speed listed here is the 2nd-order speed at $20 R_{\odot}$ taken from the LASCO online catalog.

\begin{tabular}{cccc}
\hline $\begin{array}{c}\text { Date } \\
\text { (UTC) }\end{array}$ & $\begin{array}{c}\text { Estimated GOES } \\
\text { class }\end{array}$ & AR position & $\begin{array}{c}\text { CME speed } \\
\left(\mathrm{km} \mathrm{s}^{-1}\right)\end{array}$ \\
\hline 2013 Oct 11 07:01 & M4.9 & N21E106 $\left(10^{\circ}\right.$ b.t.l $)$ & 1200 \\
2014 Jan 06 07:40 & X3.5 & S08W110 $\left(20^{\circ}\right.$ b.t.l $)$ & 1400 \\
2014 Sep 01 11:00 & X2.1 & N14E126 $\left(36^{\circ}\right.$ b.t.l $)$ & 2000 \\
\hline
\end{tabular}

the-limb solar flares were observed revealing very different properties. As such a definitive scenario for this class of flares has yet to be found. The first occulted Solar flare with both prompt and delayed line emission was observed on 1989 September 29 by GRS on-board SMM. Vestrand \& Forrest (1993) reported intense gamma-ray line emission in the 1$8 \mathrm{MeV}$ range and a strong $2.23 \mathrm{MeV}$ neutron capture line from this flare whose AR was estimated to be $15^{\circ}$ behind the western limb. Given the strength of the line emission it was concluded that a spatially extended component was required in order to explain the observations. The 1991 June 1 flare detected by PHEBUS on GRANAT had intense gamma-ray line emission in the 1-8 MeV range but no $2.2 \mathrm{MeV}$ neutron capture line detected, therefore Barat et al. (1994) concluded that the emission was of Coronal origin given the lack of a neutron capture line. In the case of the electron dominated 1991 June 30 occulted flare significant emission up to almost $100 \mathrm{MeV}$ was detected by PHEBUS, BATSE and EGRET however no line emission was reported. Vilmer et al. (1999) report that the spectral properties of this flare shared similarities with thick target emission on the visible disk however conclusive evidence in favor of this given interpretation was lacking.

The Fermi-LAT observations have allowed the sample of occulted flares to double from three to six and provide the first detections of emission in the $\mathrm{GeV}$ range from such rare events. The LAT observations sample flares from ARs originating from behind both the eastern and western limbs, they are all associated with very fast Coronal Mass Ejections (CMEs) and strong SEP events such as the event of 2014 January 6.

Table 1 lists the start times, estimated GOES classes, AR locations, and CME speeds for the three behind-the-limb flares detected by Fermi-LAT. The location of the AR was confirmed behind the limb by the STEREO spacecraft. Figure 2 shows, for the three different flares, SDO and STEREO images of the disk of the Sun as well as the location of the AR and, when possible, the centroid of the $>100 \mathrm{MeV}$ LAT emission.

For the 2013 October 11 flare the location of the AR was approximately $10^{\circ}$ behind the eastern limb. RHESSI and Fermi-GBM detected emission up to $\sim 100 \mathrm{keV}$. The location of the X-ray emission derived from RHESSI is soft and compatible with a loop top emission extending above the limb. Detailed analysis has been published in PesceRollins et al. (2015). For the 2014 September flare, the location of the AR is $\sim 20^{\circ}$ behind the western limb. This event is also interesting because it is associated with a very strong SEP event with GOES protons measured up to $700 \mathrm{MeV}$. The LAT was in the SAA until 07:55 UT therefore missing the first $\sim 10$ minutes of the flare. Upon exiting, the LAT detected gamma-ray emission from this flare for 20 minutes. Unfortunately the statistics were not sufficient to provide an emission localization error circle smaller than $0.5^{\circ}$. The last event ( 2014 September 1), on the other hand, showed a bright LAT emission lasting $\sim 2$ hours and emission up to tens of $\mathrm{MeV}$ in the GBM-BGO detectors. We also detect 

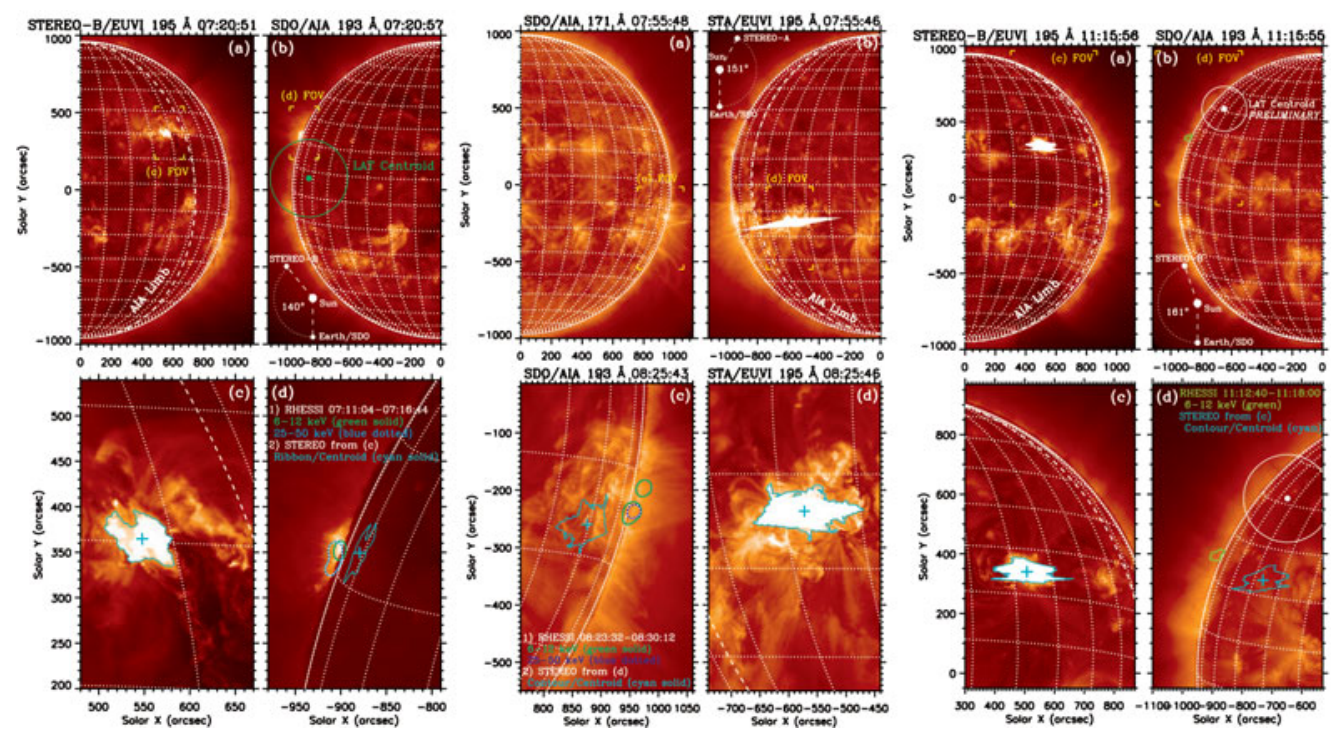

Figure 2. PRELIMINARY: The three behind the limb flares observed by Fermi-LAT. For the first flare (2013 October) and the third flare (2014 September) panel (a) is the $195 \AA$ STEREO B image and panel (a) is the $193 \AA$ from SDO/AIA, while for the second flare (2014 January) panel (a) is the $193 \AA$ from SDO/AIA and panel (b) is the $195 \AA$ STEREO A. The location of the satellite relative to the Sun is illustrated in each (b) panel. The second row shows a zoom of the ARs: the ribbon contours of the behind the limb flares are also projected on the visible side of the disc. The dashed line corresponds to the limb of the Sun as viewed from Earth and, when possible, we also draw the centroid of the LAT emission with its $68 \%$ error, for the time integrated emission.

the $2.23 \mathrm{MeV}$ neutron capture line from this flare during the peak intensity. Interestingly, the location of the AR as derived by STEREO B is $\sim 36^{\circ}$ behind the eastern limb, making this flare extremely useful for constraining the emission model.

\section{Constraining Emission Scenarios}

The high-energy emission of the 2010 June 12 solar flare (Ackermann et al. 2012) seems to be temporally correlated with the impulsive Hard X-rays (HXR) emission, suggesting that acceleration of particles and $\gamma$-ray emission takes place close in space. Specifically, particles accelerated at the loop top could propagate along the loop field lines interacting and emitting $\gamma$-rays at the footprint. For this flare there is no evidence of sustained emission suggesting long trapping time or continuous acceleration.

On the other hand, flares with long (or sustained) gamma-ray emission have also been observed by the Fermi-LAT. Temporal and spectral analysis suggests that the sustained long lasting emission is more correlated with SEP properties, indicating that, for this class of flares, either long trapping, continuous acceleration, or acceleration at the CME shock could be a better explanation (Ramaty et al. 1987; Cliver et al. 1993; Ryan 2000).

The behind-the-limb flare detection at high-energy adds additional considerations that are extremely useful for understanding the physics of particle acceleration and $\gamma$-ray production during solar flares. The $\gamma$-ray spectrum is curved, suggesting that the emission detected by the LAT originates from accelerated protons interacting with the dense regions of the Sun close to the photosphere producing pions and in turn $\gamma$-rays. Electrons can also produce high-energy $\gamma$-rays by bremsstrahlung radiation, but their energy 
distribution has to be fine tuned in order to reproduce the spectrum that is naturally produced by pion decay.

We consider three scenarios for the emission site of the $\gamma$-rays, outlined in Figure 3 . High-energy electrons or protons can produce $>100 \mathrm{MeV}$ photons after traversing a column depth which is much larger than the depth penetrated by electron producing HXR (model a). For occulted flares, the emitted photons must traverse even larger depths and they may be scattered and absorbed before exiting the Sun. Alternatively, acceleration and gammaray emission can take place in the Corona above the limb (model b). To this end, particles need to remain trapped by strongly converging magnetic fields. In the third model (model

c) CME-shock accelerated particles can travel back to the Sun along magnetic field lines connecting the acceleration site with the visible side of the Sun. Fermi-LAT observation of the

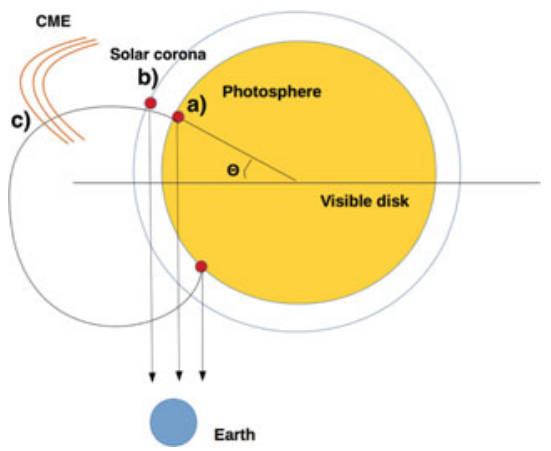

Figure 3. Model a): acceleration at the flare, gamma-ray emission site below the photosphere; b) acceleration at the flare, gamma-ray emission in the Corona above the limb; c) acceleration (or re-acceleration) at the CME-shock, gamma-ray emission at the Sun.

2013 October 11 flare shows with detailed calculations (Pesce-Rollins et al. 2015) that model a) can be ruled out as the column depth that photons have to traverse is, by far, too large. The LAT detection of gamma-ray emission from flares with $\theta>20^{\circ}$ also poses some complications to the second scenario (model b), as accelerated particles will have to produce $\gamma$-rays even higher in the Corona in region where densities are very low $\left(<10^{11} \mathrm{~cm}^{-3}\right)$. Acceleration (or re-acceleration) at the CME shock (model c) remains possible. We tentatively conclude that, high-energy emission during the impulsive phase in solar flares seems to be driven by fast precipitation of particles in proximity to the footpoints of the AR. This naturally explains the temporal correlation with HXR observed in the 2010 June 12 flare and in the impulsive phase of 2012 March 7. In this scenario, particles have access to closed field lines associated with the main loop structure, explaining why the localization of the $>100 \mathrm{MeV}$ gamma rays in 2012 March 7 flare coincides with the AR. Sustained emission is probably initiated by CME shock, as suggested by the correlation with observed SEP at Earth (Ramaty et al. 1987; Ryan 2000). Moreover, transport of charged particles along magnetic field lines in the Corona comprehends trapping, scattering and diffusion processes: depending on the details of the injection (i.e., distance from the Sun, magnetic field configuration) particles might gain access to open field lines and reach the interplanetary space and, in some cases, the Earth. In these cases an increase of the SEP flux is observed minutes/hours after the main flaring event. It is possible that, if the acceleration takes place somewhere in the Corona (by the CME shock, but not too far from the Sun), large closed lines above the loop could still trap particles and serve as a path toward the visible side of the Sun, where they will eventually precipitate producing $\gamma$-rays. Continuous acceleration processes, such as stochastic acceleration (see, for example Petrosian \& Liu 2004) might also play an important role.

\section{Conclusions}

Fermi-LAT observations are an important tool to disentangle models of particle acceleration and gamma-ray production in solar flares. The LAT localization is not accurate 
enough to precisely map the location and the extension of the high-energy $\gamma$-ray emission, in fact, during the bright solar flare of 2012 March 7, we were only able to observe a scatter of the localization across the solar disk when we split the events in separate time intervals. The behind-the-limb flares phenomenology naturally masks the impulsive phase of the flares, offering the possibility to directly observe only those particles that travel enough to cross the limb of the Sun. Finally, we suggest that these observations could be used to constrain acceleration, trapping, scattering and diffusion phenomena by comparing predictions of different models and simulations with the arrival times, duration, spectra and localization derived from Fermi-LAT observations. For example: the arrival time of the SEP is related to their time-of-flight including propagation and diffusion along the interplanetary magnetic field (Kelly et al. 2012) and the temporal details of the gamma-ray emission are related to the diffusion and propagation in the Corona. Comparing the SEP and the $\gamma$-ray light curves can help for example, constraining the altitude at which the injection took place. Observations of behind-the-limb flares also offer an important tool to study particle propagation and diffusion in the Corona, providing important constraints on the diffusion coefficient and on the configuration of large magnetic field loops. Future LAT observations, combined with a systematic study of high-energy solar flares, and with an ongoing modeling effort will very likely help to understand this fascinating problem, and to improve our knowledge of particle acceleration, diffusion, and propagation in astrophysical sources in general.

\section{Acknowledgements}

The Fermi LAT Collaboration acknowledges support from a number of agencies and institutes for both development and the operation of the LAT as well as scientific data analysis. These include NASA and DOE in the United States, CEA/Irfu and IN2P3/CNRS in France, ASI and INFN in Italy, MEXT, KEK, and JAXA in Japan, and the K. A. Wallenberg Foundation, the Swedish Research Council and the National Space Board in Sweden. Additional support from INAF in Italy and CNES in France for science analysis during the operations phase is also gratefully acknowledged.

\section{References}

Abdo, A. A., Ackermann, M., Ajello, M., et al. 2011a, ApJ, 734, 116

-. 2011b, ApJ, 734, 116

Ackermann, M., Ajello, M., Allafort, A., et al. 2012, ApJ, 745, 144

Ackermann, M., Ajello, M., Albert, A., et al. 2014, The Astrophysical Journal, 787, 15

Ajello, M. A. A., Allafort, A., Baldini, L., et al. 2014, The Astrophysical Journal, 789, 20

Atwood, W. B.Abdo, A. A., Ackermann, M., Ajello, M., et al. 2009, ApJ, 697, 1071

Barat, C., Trottet, G., Vilmer, N., et al. 1994, ApJ, 425, L109

Cliver, E. W., Kahler, S. W., \& et al. 1993, in International Cosmic Ray Conference, Vol. 3, International Cosmic Ray Conference, $91-+$

Kelly, J., Dalla, S., \& Laitinen, T. 2012, ApJ, 750, 47

Meegan, C., Lichti, G., Bhat, P. N., et al. 2009, ApJ, 702, 791

Nitta, N. V., Aschwanden, M. J., Boerner, P. F., et al. 2013, Sol. Phys., 288, 241

Pesce-Rollins, M., Omodei, N., Petrosian, V., et al. 2015, ApJ, 805, L15

Petrosian, V., \& Liu, S. 2004, ApJ, 610, 550

Ramaty, R., Murphy, R. J., \& Dermer, C. D. 1987, ApJ, 316, L41

Ryan, J. M. 2000, Space Sci. Rev., 93, 581

Vestrand, W. T., \& Forrest, D. J. 1993, ApJ, 409, L69

Vilmer, N., Trottet, G., Barat, C., et al. 1999, A\&A, 342, 575 